\title{
Radiative transfer of elastic waves in two-dimensional isotropic scattering media: Semi-analytical approach for isotropic source radiation
}

\author{
Hisashi Nakahara $^{1}$ and Kazuo Yoshimoto ${ }^{2}$ \\ ${ }^{1}$ Department of Geophysics, Graduate School of Science, Tohoku University, \\ 6-3 Aramaki-Aza-Aoba, Aoba-ku, Sendai 980-8578, Japan \\ ${ }^{2}$ Graduate School of Nanobioscience, International College of Arts and Science, Basic and Applied Sciences, \\ Yokohama City University, 22-2 Seto, Kanazawa-ku, Yokohama 236-0027, Japan
}

(Received October 14, 2010; Revised February 1, 2011; Accepted March 16, 2011; Online published September 7, 2011)

\begin{abstract}
We formulate the radiative transfer of $P$ - and $S$-wave energy from an isotropically radiating source in a twodimensional infinite isotropic scattering medium. For a stable numerical calculation of seismogram envelopes, we take a semi-analytical approach: Energy densities of $P$ and $S$ waves are divided into three parts of the directwave terms, the single-scattering terms, and the multiple-scattering terms, and the first two terms and the last term are evaluated analytically and numerically, respectively. Concerning the single-scattering terms, the $P$ to- $S$ and $S$-to- $P$ single conversion scattering terms are expressed analytically with a complete elliptic integral of the first kind. The multiple-scattering terms are represented by a double integral with respect to frequency and wavenumber, and can be numerically evaluated by a discrete wavenumber summation and a Fast Fourier Transform. The results based on the numerical implementation are confirmed with an independent numerical calculation using the Monte Carlo method. Our formulation is also applied to consider the equilibration between $P$ and $S$ waves at larger lapse times. The equilibrated $S$-to- $P$ energy ratio is reproduced and the equilibration time is first derived for two-dimensional cases. Our formulation will be a reference for the understanding of more complex cases.
\end{abstract}

Key words: Radiative transfer, two-dimensional, $S$-to- $P$ energy ratio, equilibration.

\section{Introduction}

Seismogram envelopes have been used to estimate the strength of scattering and absorption in the Earth. Synthesizing realistic seismogram envelopes is needed to model observed envelopes and to invert for the strength of scattering and absorption in the Earth. Radiative transfer theory, which was originally developed in astrophysics (e.g. Chandrasekhar, 1960), is widely used in seismology, because it offers clear physical meanings and mathematical tractability. Various kinds of settings have been modeled using radiative transfer theory in seismology. For more detailed developments, readers can refer to Sato and Fehler (1998). Modeling started from the single scattering of scalar waves for an isotropic source and isotropic scattering in three-dimensional media (e.g. Aki and Chouet, 1975; Sato, 1977a), and in two-dimensional media (e.g. Kopnichev, 1975). Later, multiple scattering was included in the modeling for the three-dimensional case by $\mathrm{Wu}$ (1985) and Zeng et al. (1991), and for the two-dimensional case by Shan and Gao (1988). Vector waves (or $P$ waves and $S$ waves) were first considered for the single-scattering case by Sato (1977b) and for the multiple scattering case by Zeng (1993) and Sato (1994), all of which treated threedimensional cases. However, concerning two-dimensional

Copyright (c) The Society of Geomagnetism and Earth, Planetary and Space Sciences (SGEPSS); The Seismological Society of Japan; The Volcanological Society of Japan; The Geodetic Society of Japan; The Japanese Society for Planetary Sciences; TERRAPUB.

doi:10.5047/eps.2011.03.006 cases, we have only found a paper by Trégourès and van Tiggelen (2002) which derived a radiative transfer equation in a thin-plate bounded by two free surfaces. A canonical problem for a two-dimensional full space has not been found.

The partitioning of $P$ waves and $S$ waves is an important subject. The $S$-to- $P$ energy ratio is known to be equilibrated after multiple conversion scatterings between $P$ waves and $S$ waves. The equilibrated ratio was elegantly derived for three-dimensional full spaces by Weaver (1982), and, later, for two-dimensional full spaces by SánchezSesma and Campillo (2006). To consider the transition process into the equilibrium, radiative transfer theory for vector waves is necessary; see Zeng (1993), Sato (1994) and Ryzhik et al. (1996). Snieder (2002) proposed a simpler ball-counting method. The $S$-to- $P$ energy ratio can be used to judge whether a wavefield is in a diffuse state (multiple scattering regime) or not. Shapiro et al. (2000) conducted the first seismic observation in this context and concluded that seismic coda is in a diffuse state. Sánchez-Sesma and Campillo (2006) clarified that the $S$-to- $P$ energy ratio is also important to retrieve strict Green's tensors from crosscorrelations of noise records using seismic interferometry, which is a rapidly-growing field in seismology, acoustics, exploration geophysics, etc. (e.g. Curtis et al., 2006).

In this study, we formulate the radiative transfer of elastic waves in a two-dimensional full space for the first time. Simple assumptions on the isotropy of source radiation and scattering enable us to derive semi-analytical expressions: 
the direct-wave terms and the single-scattering terms are analytically evaluated, and the multiple-scattering terms having an order higher than or equal to two are numerically calculated. The numerical implementation of our formulation is tested and confirmed by comparisons with the purely numerical approach of a Monte Carlo simulation. Finally, we discuss the energy partitioning of a $P$ wave and an $S$ wave in two-dimensional media as an application of our formulation.

\section{Formulation}

\subsection{Basic equations}

We assume that $P$-wave energy $W^{P}$ and $S$-wave energy $W^{S}$ are impulsively radiated from an isotropic source located at the origin in a two-dimensional isotropic scattering medium (Fig. 1). Strictly speaking, assumptions of isotropic source radiation and an isotropic scattering pattern are too simple. But they greatly help to understand the mathematical background and to provide a reference for more realistic (or complex) cases. On the way to receivers, energy is scattered and converted from $P$-to- $S$ and $S$-to- $P$. Energy densities for $P$ waves and $S$ waves at location $\mathbf{x}$ and time $t$, which are denoted as $E^{P}(\mathbf{x}, t)$ and $E^{S}(\mathbf{x}, t)$, satisfy the following integral equation (e.g. Sato, 1994):

$$
\begin{aligned}
E^{P}(\mathbf{x}, t)= & W^{P} G^{P}(\mathbf{x}, t)+\int_{-\infty}^{\infty} \int_{-\infty}^{\infty} \int_{-\infty}^{\infty}\left\{E^{P}\left(\mathbf{x}^{\prime}, t^{\prime}\right) \alpha_{0} g_{0}^{P P}\right. \\
& \left.+E^{S}\left(\mathbf{x}^{\prime}, t^{\prime}\right) \beta_{0} g_{0}^{S P}\right\} G^{P}\left(\mathbf{x}-\mathbf{x}^{\prime}, t-t^{\prime}\right) d t^{\prime} d \mathbf{x}^{\prime},(1) \\
E^{S}(\mathbf{x}, t)= & W^{S} G^{S}(\mathbf{x}, t)+\int_{-\infty}^{\infty} \int_{-\infty}^{\infty} \int_{-\infty}^{\infty}\left\{E^{S}\left(\mathbf{x}^{\prime}, t^{\prime}\right) \beta_{0} g_{0}^{S S}\right. \\
& \left.+E^{P}\left(\mathbf{x}^{\prime}, t^{\prime}\right) \alpha_{0} g_{0}^{P S}\right\} G^{S}\left(\mathbf{x}-\mathbf{x}^{\prime}, t-t^{\prime}\right) d t^{\prime} d \mathbf{x}^{\prime},(2)
\end{aligned}
$$

where $\alpha_{0}, \beta_{0}$ are, respectively, the $P$-wave and $S$-wave velocity, and $g_{0}^{P P}, g_{0}^{P S}, g_{0}^{S P}, g_{0}^{S S}$ are total scattering coefficients for $P$-to- $P, P$-to- $S, S$-to- $P, S$-to- $S$ scattering. $G^{P}(\mathbf{x}, t)$ is a propagator for $P$-wave energy which is characterized by the geometrical spreading factor of a reciprocal of $r=|\mathbf{x}|$ and propagation with $P$-wave velocity (e.g. Shang and Gao, 1988; Sato, 1993) as:

$$
G^{P}(\mathbf{x}, t)=\frac{1}{2 \pi \alpha_{0} r} H(t) \delta\left(t-\frac{r}{\alpha_{0}}\right) e^{-\left(\alpha_{0} g_{0}^{P P}+\alpha_{0} g_{0}^{P S}+b\right) t}
$$

Similarly, $G^{S}(\mathbf{x}, t)$ is a propagator for $S$-wave energy as:

$$
G^{S}(\mathbf{x}, t)=\frac{1}{2 \pi \beta_{0} r} H(t) \delta\left(t-\frac{r}{\beta_{0}}\right) e^{-\left(\beta_{0} g_{0}^{S S}+\beta_{0} g_{0}^{S P}+b\right) t},
$$

where $b$ is an intrinsic absorption coefficient which is assumed to be equal for both $P$ and $S$ waves here. Operating a Fourier transform with respect to space and a Laplace transform with respect to time on Eqs. (3) and (4), we can

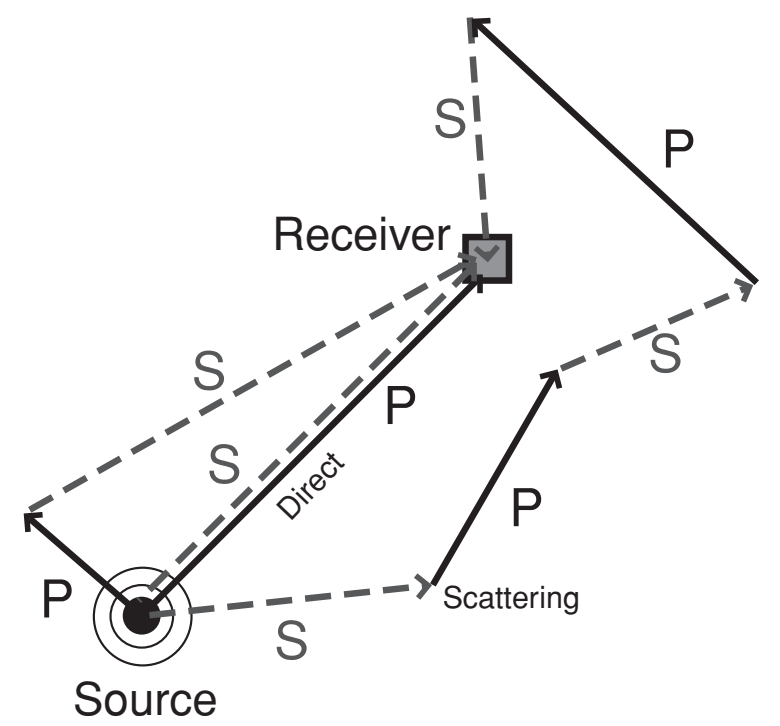

Fig. 1. Schematic illustration of $P$-wave and $S$-wave energy propagation in a two-dimensional isotropic scattering medium. A source and a receiver are shown by a concentric circle and a gray rectangle. Propagation as a $P$ wave and that as an $S$ wave are shown by solid arrows and gray broken arrows, respectively. $P$-wave and $S$-wave energy which is radiated from an isotropic source is scattered and converted between $P$ wave and $S$ wave on the way to a receiver.

get the following two relations:

$$
\begin{aligned}
\hat{\tilde{G}}^{P}(k, s) & \equiv \int_{-\infty}^{\infty} \int_{-\infty}^{\infty} d \mathbf{x} e^{-i \mathbf{k} \cdot \mathbf{x}} \int_{0}^{\infty} d t e^{-s t} G^{P}(\mathbf{x}, t) \\
& =\frac{1}{\sqrt{\left(s+\alpha_{0} g_{0}^{P P}+\alpha_{0} g_{0}^{P S}+b\right)^{2}+\left(\alpha_{0} k\right)^{2}}}, \\
\hat{\tilde{G}}^{S}(k, s) & \equiv \int_{-\infty}^{\infty} \int_{-\infty}^{\infty} d \mathbf{x} e^{-i \mathbf{k} \cdot \mathbf{x}} \int_{0}^{\infty} d t e^{-s t} G^{S}(\mathbf{x}, t) \\
& =\frac{1}{\sqrt{\left(s+\beta_{0} g_{0}^{S S}+\beta_{0} g_{0}^{S P}+b\right)^{2}+\left(\beta_{0} k\right)^{2}}},
\end{aligned}
$$

where ^ means Laplace transform, $\sim$ means Fourier transform, and $k=|\mathbf{k}|$.

The Fourier-Laplace transform of Eqs. (1) and (2) leads to the following two equations:

$$
\hat{\tilde{E}}^{P}(k, s)=\frac{W^{P} \hat{\tilde{G}}^{P}\left(1-\beta_{0} g_{0}^{S S} \hat{\tilde{G}}^{S}\right)+W^{S} \hat{\tilde{G}}^{S} \beta_{0} g_{0}^{S P} \hat{\tilde{G}}^{P}}{\left(1-\alpha_{0} g_{0}^{P P} \hat{\tilde{G}}^{P}\right)\left(1-\beta_{0} g_{0}^{S S} \hat{\tilde{G}}^{S}\right)-\alpha_{0} g_{0}^{P S} \beta_{0} g_{0}^{S P} \hat{\tilde{G}}^{P} \hat{\tilde{G}}^{S}},
$$

$$
\hat{\tilde{E}}^{S}(k, s)=\frac{W^{S} \hat{\tilde{G}}^{S}\left(1-\alpha_{0} g_{0}^{P P} \hat{\tilde{G}}^{P}\right)+W^{P} \hat{\tilde{G}}^{P} \alpha_{0} g_{0}^{P S} \hat{\tilde{G}}^{S}}{\left(1-\alpha_{0} g_{0}^{P P} \hat{\tilde{G}}^{P}\right)\left(1-\beta_{0} g_{0}^{S S} \hat{\tilde{G}}^{S}\right)-\alpha_{0} g_{0}^{P S} \beta_{0} g_{0}^{S P} \hat{\tilde{G}}^{P} \hat{\tilde{G}}^{S}} .
$$

These are the formal solutions in the wavenumber and frequency domain. However, the direct inverse FourierLaplace transform of Eqs. (7) and (8) asks for careful numerical treatments, as pointed out by Zeng (1993). So we take the different approach proposed by Sato (1994). We divide the total energy density into a direct wave part, a single-scattering part, and a multiple-scattering part having 
an order of higher than or equal to 2 as shown in Eq. (9) for $P$ waves:

$$
\hat{\tilde{E}}^{P}(k, s)=\hat{\tilde{E}}^{P, 0}(k, s)+\hat{\tilde{E}}^{P, 1}(k, s)+\hat{\tilde{E}}^{P, M}(k, s) .
$$

The direct $P$ wave $\hat{\tilde{E}}^{P, 0}(k, s)$ is radiated from the source as a $P$ wave and reaches the receiver without any scattering:

$$
\hat{\tilde{E}}^{P, 0}(k, s)=W^{P} \hat{\tilde{G}}^{P} .
$$

The single scattering term $\hat{\tilde{E}}^{P, 1}(k, s)$ is composed of two parts:

$$
\hat{\tilde{E}}^{P, 1}(k, s)=W^{P} \hat{\tilde{G}}^{P} \alpha_{0} g_{0}^{P P} \hat{\tilde{G}}^{P}+W^{S} \hat{\tilde{G}}^{S} \beta_{0} g_{0}^{S P} \hat{\tilde{G}}^{P} .
$$

On the right-hand side, the first term is a $P$ wave which is radiated from the source as a $P$ wave, scattered once into a $P$ wave, and reaches the receiver. The second term is a $P$ wave which is radiated from the source as an $S$ wave, scattered once into a $P$ wave, and reaches the receiver.

The last term $\hat{\tilde{E}}^{P, M}(k, s)$ is the summation of energy which is radiated from the source as a $P$ wave or an $S$ wave and scattered more than twice and reaches the receiver as a $P$ wave:

$$
\begin{aligned}
\hat{\tilde{E}}^{P, M}(k, s)= & \frac{1}{\left(1-\alpha_{0} g_{0}^{P P} \tilde{\tilde{G}}^{P}\right)\left(1-\beta_{0} g_{0}^{S S} \tilde{\tilde{G}}^{S}\right)-\alpha_{0} g_{0}^{P S} \beta_{0} g_{0}^{S P} \tilde{\tilde{G}}^{P} \hat{\tilde{G}}^{S}} \\
& \times\left(W ^ { P } \hat { \tilde { G } } ^ { P } \left\{\left(\alpha_{0} g_{0}^{P P} \hat{\tilde{G}}^{P}\right)^{2}\left(1-\beta_{0} g_{0}^{S S} \hat{\tilde{G}}^{S}\right)\right.\right. \\
& \left.+\alpha_{0} g_{0}^{P S} \beta_{0} g_{0}^{S P}\left(1+\alpha_{0} g_{0}^{P P} \hat{\tilde{G}}^{P}\right) \tilde{\tilde{G}}^{P} \hat{\tilde{G}}^{S}\right\} \\
& +W^{S} \hat{\tilde{G}}^{S}\left\{\beta _ { 0 } g _ { 0 } ^ { S P } \hat { \tilde { G } } ^ { P } \left[\alpha_{0} g_{0}^{P P} \hat{\tilde{G}}^{P}\left(1-\beta_{0} g_{0}^{S S} \hat{\tilde{G}}^{S}\right)\right.\right. \\
& \left.\left.\left.+\beta_{0} g_{0}^{S S} \hat{\tilde{G}}^{S}+\alpha_{0} g_{0}^{P S} \beta_{0} g_{0}^{S P} \hat{\tilde{G}}^{P} \hat{\tilde{G}}^{S}\right]\right\}\right) .
\end{aligned}
$$

The corresponding expressions for $S$ waves are as follows:

$$
\begin{aligned}
\hat{\tilde{E}}^{S}(k, s) & =\hat{\tilde{E}}^{S, 0}(k, s)+\hat{\tilde{E}}^{S, 1}(k, s)+\hat{\tilde{E}}^{S, M}(k, s),(13) \\
\hat{\tilde{E}}^{S, 0}(k, s) & =W^{S} \hat{\tilde{G}}^{S}, \\
\hat{\tilde{E}}^{S, 1}(k, s) & =W^{S} \hat{\tilde{G}}^{S} \beta_{0} g_{0}^{S S} \hat{\tilde{G}}^{S}+W^{P} \hat{\tilde{G}}^{P} \alpha_{0} g_{0}^{P S} \hat{\tilde{G}}^{S},(15) \\
\hat{\tilde{E}}^{S, M}(k, s)= & \frac{1}{\left(1-\alpha_{0} g_{0}^{P P} \hat{\tilde{G}}^{P}\right)\left(1-\beta_{0} g_{0}^{S S} \hat{\tilde{G}}^{S}\right)-\alpha_{0} g_{0}^{P S} \beta_{0} g_{0}^{S P} \hat{\tilde{G}}^{P} \hat{\tilde{G}}^{S}} \\
& \times\left(W ^ { S } \tilde { \tilde { G } } ^ { S } \left\{\left(\beta_{0} g_{0}^{S S} \hat{\tilde{G}}^{S}\right)^{2}\left(1-\alpha_{0} g_{0}^{P P} \hat{\tilde{G}}^{P}\right)\right.\right. \\
& \left.+\alpha_{0} g_{0}^{P S} \beta_{0} g_{0}^{S P}\left(1+\beta_{0} g_{0}^{S S} \hat{\tilde{G}}^{S}\right) \hat{\tilde{G}}^{P} \hat{\tilde{G}}^{S}\right\} \\
& +W^{P} \hat{\tilde{G}}^{P}\left\{\alpha _ { 0 } g _ { 0 } ^ { P S } \hat { \tilde { G } } ^ { S } \left[\beta_{0} g_{0}^{S S} \hat{\tilde{G}}^{S}\left(1-\alpha_{0} g_{0}^{P P} \hat{\tilde{G}}^{P}\right)\right.\right. \\
& \left.\left.\left.+\alpha_{0} g_{0}^{P P} \hat{\tilde{G}}^{P}+\alpha_{0} g_{0}^{P S} \beta_{0} g_{0}^{S P} \hat{\tilde{G}}^{P} \hat{\tilde{G}}^{S}\right]\right\}\right)
\end{aligned}
$$

We assume the same attenuation for both $P$ and $S$ waves as:

$$
\alpha_{0} g_{0}^{P P}+\alpha_{0} g_{0}^{P S}+b=\beta_{0} g_{0}^{S S}+\beta_{0} g_{0}^{S P}+b=\eta .
$$

This is a requirement for the analytical derivation of the single-scattering terms as shown in the next subsection and Appendix. The observed ratio of the attenuation of a $P$ wave to that of an $S$ wave for frequencies higher than $1 \mathrm{~Hz}$ mostly ranges between 0.7 and 2 in the lithosphere as shown in figure 5.3 of Sato and Fehler (1998). Therefore, Eq. (17) might be the first-order approximation. However, when this assumption does not hold in a strict sense, we need to make an inverse Fourier-Laplace transform of Eqs. (7) and (8) directly.

\subsection{Analytical representation of the single-scattering terms}

In principle, it is possible to estimate the single-scattering terms both by integration in the space-time domain and the inverse Fourier-Laplace transform in the wavenumberfrequency domain. We can obtain $P$-to- $P$ and $S$-to- $S$ single scatterings by both methods (e.g. Sato, 1993). However, concerning $P$-to- $S$ and $S$-to- $P$ single conversion scatterings, we have so far only succeeded in deriving the analytical expressions by integration in the space-time domain using elliptical coordinates (e.g. page 46 in Sato and Fehler, 1998; page 1195 in Morse and Feshbach, 1953). Here, we briefly summarize the results. The detailed derivation is shown in Appendix.

Four terms of $P$-to- $P, P$-to- $S, S$-to- $P, S$-to- $S$ singlescattering energy densities are denoted as $E^{P P, 1}(\mathbf{x}, t)$, $E^{P S, 1}(\mathbf{x}, t), E^{S P, 1}(\mathbf{x}, t), E^{S S, 1}(\mathbf{x}, t)$ respectively. The $P$ to- $P$ single-scattering term is expressed as:

$$
E^{P P, 1}(\mathbf{x}, t)=\frac{W^{P} g_{0}^{P P}}{2 \pi r} \frac{H\left(\frac{\alpha_{0} t}{r}-1\right) e^{-\eta t}}{\sqrt{\left(\frac{\alpha_{0} t}{r}\right)^{2}-1}} .
$$

This single-scattering term is equal to the scalar wave case with $P$-wave velocity (e.g. Sato, 1993 ). The $S$-to- $S$ singlescattering term can be derived similarly as:

$$
E^{S S, 1}(\mathbf{x}, t)=\frac{W^{S} g_{0}^{S S}}{2 \pi r} \frac{H\left(\frac{\beta_{0} t}{r}-1\right) e^{-\eta t}}{\sqrt{\left(\frac{\beta_{0} t}{r}\right)^{2}-1}} .
$$

Conversion scattering terms of $P$-to- $S$ and $S$-to- $P$ are more complex:

$$
E^{P S, 1}(\mathbf{x}, t)=\frac{W^{P} g_{0}^{P S} \gamma}{\pi^{2} r} M\left(\frac{\alpha_{0} t}{r}\right) H\left(\frac{\alpha_{0} t}{r}-1\right) e^{-\eta t},
$$

where

$$
\begin{aligned}
M(x) & \equiv \frac{1}{\sqrt{\gamma^{2}-1}} K\left(\sqrt{\frac{x^{2}-1}{\gamma^{2}-1}}\right) \quad \text { for } \quad 1 \leq x<\gamma \\
& =\frac{1}{\sqrt{x^{2}-1}} K\left(\sqrt{\frac{\gamma^{2}-1}{x^{2}-1}}\right) \quad \text { for } \quad x \geq \gamma,
\end{aligned}
$$


and

$$
\gamma \equiv \frac{\alpha_{0}}{\beta_{0}}
$$

and $K(x)$ is the complete elliptic integral of the first kind (e.g. page 590 in Abramowitz and Stegun, 1970). It is noted that this function also appears in the single scattering of scalar waves on a spherical surface (Maeda et al., 2003).

We find that the $S$-to- $P$ single-scattering term has the same space-time dependence as the $P$-to- $S$ singlescattering term:

$$
\begin{aligned}
E^{S P, 1}(\mathbf{x}, t) & =\frac{W^{S} g_{0}^{S P}}{\pi^{2} r} M\left(\frac{\alpha_{0} t}{r}\right) H\left(\frac{\alpha_{0} t}{r}-1\right) e^{-\eta t} \\
& =\frac{W^{S}}{W^{P} \gamma^{2}} E^{P S, 1}(\mathbf{x}, t) .
\end{aligned}
$$

\subsection{Total energy density of $\boldsymbol{P}$ waves and $S$ waves}

Evaluating the direct-wave terms and the singlescattering terms in the space-time domain, the total energy density of $P$ waves is expressed as follows:

$$
\begin{aligned}
E^{P}(r, t)= & \frac{W^{P} e^{-\eta t}}{2 \pi \alpha_{0} r} H(t) \delta\left(t-\frac{r}{\alpha_{0}}\right) \\
& +\frac{W^{P} g_{0}^{P P} e^{-\eta t}}{2 \pi r \sqrt{\left(\frac{\alpha_{0} t}{r}\right)^{2}-1} H\left(\frac{\alpha_{0} t}{r}-1\right)} \\
& +\frac{W^{S} g_{0}^{S P} e^{-\eta t}}{\pi^{2} r} M\left(\frac{\alpha_{0} t}{r}\right) H\left(\frac{\alpha_{0} t}{r}-1\right) \\
& +\frac{1}{(2 \pi)^{2}} \int_{0}^{\infty} d k k J_{0}(k r) \int_{-\infty}^{\infty} d \omega e^{-i \omega t} \hat{\tilde{E}}^{P, M}(k, s=-i \omega) .
\end{aligned}
$$

The third term in the right-hand side corresponds to the multiple-scattering $P$ wave having an order higher than or equal to 2. This term depends only on $k$. The Bessel function of the first kind $J_{0}(k r)$ originates from a twodimensional Fourier transform. The term can be evaluated numerically using a discrete wavenumber summation (e.g. Bouchon, 1981; Zeng, 1993), equivalent to the trapezoidal rule, with respect to wavenumber, and the Fast Fourier Transform (FFT) with respect to frequency. A similar expression for $S$ waves is as follows:

$$
\begin{aligned}
& E^{S}(r, t)=\frac{W^{S} e^{-\eta t}}{2 \pi \beta_{0} r} H(t) \delta\left(t-\frac{r}{\beta_{0}}\right) \\
& +\frac{W^{S} g_{0}^{S S} e^{-\eta t}}{2 \pi r \sqrt{\left(\frac{\beta_{0} t}{r}\right)^{2}-1}} H\left(\frac{\beta_{0} t}{r}-1\right) \\
& +\frac{W^{P} g_{0}^{P S} \gamma e^{-\eta t}}{\pi^{2} r} M\left(\frac{\alpha_{0} t}{r}\right) H\left(\frac{\alpha_{0} t}{r}-1\right) \\
& +\frac{1}{(2 \pi)^{2}} \int_{0}^{\infty} d k k J_{0}(k r) \int_{-\infty}^{\infty} d \omega e^{-i \omega t} \hat{\tilde{E}}^{S, M}(k, s=-i \omega) .
\end{aligned}
$$

\subsection{Self-consistency of the formulation}

In order to check the self-consistency of the formulation, we consider the energy conservation of a $P$ wave and an $S$ wave. Substituting $k=0$ into Eq. (7) leads to:

$$
\begin{aligned}
\hat{\tilde{E}}^{P}(k=0, s) & =\int_{-\infty}^{\infty} \int_{-\infty}^{\infty} d \mathbf{x} \int_{-\infty}^{\infty} d t e^{-s t} E^{P}(\mathbf{x}, t) \\
& =\frac{\left(W^{P}+W^{S}\right) \beta_{0} g_{0}^{S P}+W^{P}(s+b)}{(s+b)\left(s+b+\alpha_{0} g_{0}^{P S}+\beta_{0} g_{0}^{S P}\right)} .
\end{aligned}
$$

The inverse Laplace transform of this equation leads to:

$$
\begin{aligned}
\tilde{E}^{P}(k= & 0, t)=\int_{-\infty}^{\infty} \int_{-\infty}^{\infty} d \mathbf{x} E^{P}(\mathbf{x}, t) \\
& =\left[\frac{\left(W^{P}+W^{S}\right) \beta_{0} g_{0}^{S P}}{\alpha_{0} g_{0}^{P S}+\beta_{0} g_{0}^{S P}}\right. \\
& \left.+\frac{W^{P} \alpha_{0} g_{0}^{P S}-W^{S} \beta_{0} g_{0}^{S P}}{\alpha_{0} g_{0}^{P S}+\beta_{0} g_{0}^{S P}} e^{-\left(\alpha_{0} g_{0}^{P S}+\beta_{0} g_{0}^{S P}\right) t}\right] e^{-b t} .
\end{aligned}
$$

Similarly,

$$
\begin{gathered}
\hat{\tilde{E}}^{S}(k=0, s)=\int_{-\infty}^{\infty} \int_{-\infty}^{\infty} d \mathbf{x} \int_{-\infty}^{\infty} d t e^{-s t} E^{S}(\mathbf{x}, t) \\
=\frac{\left(W^{P}+W^{S}\right) \alpha_{0} g_{0}^{P S}+W^{S}(s+b)}{(s+b)\left(s+b+\alpha_{0} g_{0}^{P S}+\beta_{0} g_{0}^{S P}\right)} \\
\tilde{E}^{S}(k=0, t)=\int_{-\infty}^{\infty} \int_{-\infty}^{\infty} d \mathbf{x} E^{S}(\mathbf{x}, t) \\
=\left[\frac{\left(W^{P}+W^{S}\right) \alpha_{0} g_{0}^{P S}}{\alpha_{0} g_{0}^{P S}+\beta_{0} g_{0}^{S P}}\right. \\
\left.+\frac{W^{S} \beta_{0} g_{0}^{S P}-W^{P} \alpha_{0} g_{0}^{P S}}{\alpha_{0} g_{0}^{P S}+\beta_{0} g_{0}^{S P}} e^{-\left(\alpha_{0} g_{0}^{P S}+\beta_{0} g_{0}^{S P}\right) t}\right] e^{-b t} .
\end{gathered}
$$

We find that

$$
\begin{aligned}
\tilde{E}^{P}(k=0, t)+\tilde{E}^{S}(k=0, t) & =\int_{-\infty}^{\infty} \int_{-\infty}^{\infty} d \mathbf{x}\left(E^{P}(\mathbf{x}, t)+E^{S}(\mathbf{x}, t)\right) \\
& =\left(W^{P}+W^{S}\right) e^{-b t}
\end{aligned}
$$

When $b=0$, the sum of the $P$-wave and $S$-wave energy is conserved, which means that our formulation is selfconsistent.

\section{Numerical Calculation}

\subsection{Numerical implementation of the formulation}

We evaluate the direct-wave and the single-scattering terms analytically, and the multiple-scattering terms with the discrete wavenumber summation and FFT. For numerical implementations, we introduce the following normalized (non-dimensional) quantities:

$$
\begin{aligned}
& \rho=g_{0}^{P} r, \quad \tau=g_{0}^{P} \alpha_{0} t, \\
& \varepsilon^{P}(\rho, \tau)=\frac{E^{P}(r, t)}{W^{P}\left(g_{0}^{P}\right)^{2}}, \quad \varepsilon^{S}(\rho, \tau)=\frac{E^{S}(r, t)}{W^{P}\left(g_{0}^{P}\right)^{2}},
\end{aligned}
$$


where $g_{0}^{P} \equiv g_{0}^{P P}+g_{0}^{P S}$. We need to discretize the spacetime domain. In the following, we divide the normalized space range $0 \leq \rho \leq 40.96$ with a step of 0.04 and divide the normalized time range $0 \leq \tau \leq 20.48$ with a step of 0.01. In evaluating the single-scattering terms, we use a subroutine for the function $K(x)$ in Press et al. (1992). In the discrete wavenumber summation, we should set the range where the effect of fictitious sources does not appear, as:

$$
0<\rho<\frac{\rho_{\max }}{2}, \quad \tau<\rho_{\max }-\rho
$$

$\rho_{\max }=40.96$ in this case. We assume that the damping of the medium is 0 . However, we introduce an artificial damping $b=0.5 \times g_{0}^{P} \alpha_{0}$ in the wavenumber-frequency domain for preventing numerical instabilities. The artificial damping is compensated by multiplication of $e^{b t}$ in the time domain. We assume a Poisson solid of $\alpha_{0}=6.0(\mathrm{~km} / \mathrm{s})$ and $\beta_{0}=3.46(\mathrm{~km} / \mathrm{s})$. Concerning scattering parameters, we assume $g_{0}^{P P}=g_{0}^{P S}=0.05\left(\mathrm{~km}^{-1}\right)$. Then, $g_{0}^{S P}=0.029$ $\left(\mathrm{km}^{-1}\right)$ and $g_{0}^{S S}=0.144\left(\mathrm{~km}^{-1}\right)$ are determined due to the constraints of Eqs. (17) and (A.10). The values might be larger for the usual Earth's crust, but are comparable in a volcanic region (e.g. Yamamoto and Sato, 2010). We use such larger scattering coefficients because we are interested in multiple conversion scatterings and their influence on the partitioning of $P$-wave and $S$-wave energy as discussed in the next section. For these parameters, the range of calculation in real space and time is $0 \leq r \leq 409.6[\mathrm{~km}]$, $0 \leq t \leq 34.13$ [s].

\subsection{Monte Carlo simulation to confirm the numerical implementation}

It is necessary to check the numerical implementation of our formulation. For the calculation of seismogram envelopes, a Monte Carlo simulation (e.g. Gusev and Abubakirov, 1987; Hoshiba, 1991) is versatile enough to be applicable to any type of random media and source radiation at the cost of computation time. In this study, we use a two-dimensional version of Yoshimoto (2000), which is equivalent to Przybilla et al. (2006). For the calculation, we emanate 1,000,000 particles from the source. On the way to receivers, scatterings can take place probabilistically according to the assumed medium parameters. The time step is assumed to be $0.0167 \mathrm{~s}$ from $d t=d \tau /\left(g_{0}^{P} \alpha_{0}\right)$. We count the number of particles arriving at a receiver and at a time. We can trace each particle from the source to the receiver by keeping track of the number of scatterings. So we can extract the single-scattering terms and the multiple-scattering terms separately even with the Monte Carlo method. The resultant envelopes are also normalized according to Eq. (31) for comparison with the envelopes based on our formulation.

\subsection{Results}

In Fig. 2, we show the energy densities of four singlescattering terms of $P$-to- $P, P$-to- $S, S$-to- $P, S$-to- $S$ scattering at a receiver with a normalized distance $\rho$ of 0.2 . The horizontal axis is the normalized time $\tau$ and the vertical is the normalized energy density $\varepsilon^{P}, \varepsilon^{S}$ as defined in Eq. (31). The panel shows the contribution of the four terms. Solid curves are obtained by our implementation. Gray curves

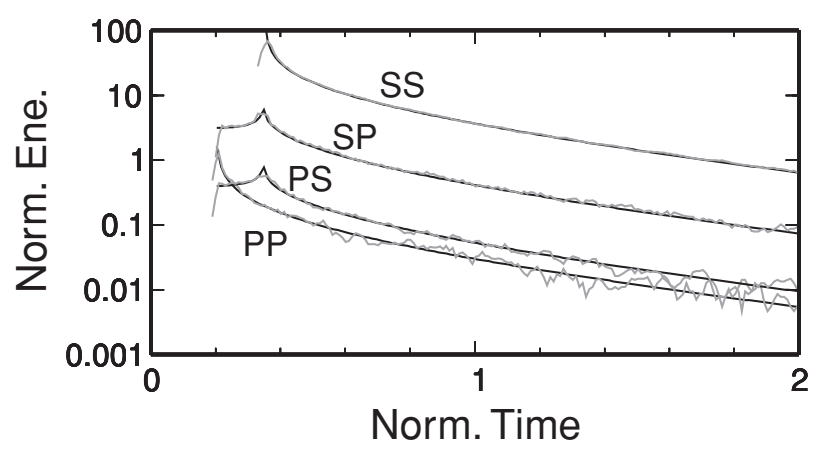

Fig. 2. Energy density of four single-scattering terms of $P$-to- $P, P$-to- $S$, $S$-to- $P, S$-to- $S$ scatterings at a receiver with a normalized distance of 0.2 . The horizontal axis is the normalized time and the vertical is the normalized energy density as defined in Eq. (31). Solid curves are produced by our implementation. Gray curves are calculated based on the Monte Carlo method for comparison.

are calculated based on the Monte Carlo simulation. Both traces show good coincidence with each other. Because $W^{S}=1.5 \gamma^{5} W^{P}=23.38 W^{P}$ assuming a Poisson solid of $\gamma=\sqrt{3}, S$-to- $S$ and $S$-to- $P$ single-scattering terms are larger than the other two terms. We also note that $S$-to- $P$ and $P$-to- $S$ single-scattering terms are similar as proved in Eq. (23). The $P$-to- $P$ and $S$-to- $S$ scattering terms have a square-root singularity at the onset times of $P$ waves and $S$ waves, respectively. The $P$-to- $S$ and $S$-to- $P$ scattering terms have a logarithmic singularity at the onset of an $S$ wave. Both kinds of singularities are integrable. The Monte Carlo results show larger fluctuations at larger lapse times especially for a $P$ wave due to the smaller number of $P$ wave particles. In Fig. 3, we show the contribution of single scattering (dotted curves) and multiple scattering (broken curves) in the total energy density (gray solid curves) at two receivers with the normalized distances of 1 and 2 . The top panel shows an $S$ wave and the bottom panel shows a $P$ wave. The single-scattering term is dominant around the onset times of a $P$ wave and an $S$ wave. On the other hand, the multiple-scattering term grows soon after the $P$-wave onset. A small fluctuation in $S$-wave energy at lapse times between $P$-wave onset and $S$-wave onset is found. This is due to numerical instabilities which we cannot so far remove. However, we think that the fluctuation is not serious because its amplitude is less than $1 \%$ of the maximum amplitude of the $S$-wave energy.

In Fig. 4, we show temporal changes of $P$-wave and $S$-wave energy density at two receivers with normalized distances of 1 and 2. All of the direct-wave, the singlescattering and the multiple-scattering terms are included. Solid curves are from our implementation. Gray curves are calculated by the Monte Carlo method. Both traces show fairly good matches. This provides confirmation that we can correctly calculate $P$-wave and $S$-wave energy density by our proposed method.

\section{Discussions}

We have succeeded in formulating the elastic radiative transfer for a two-dimensional random full space for the first time. This is a canonical problem. It is not easy to find 

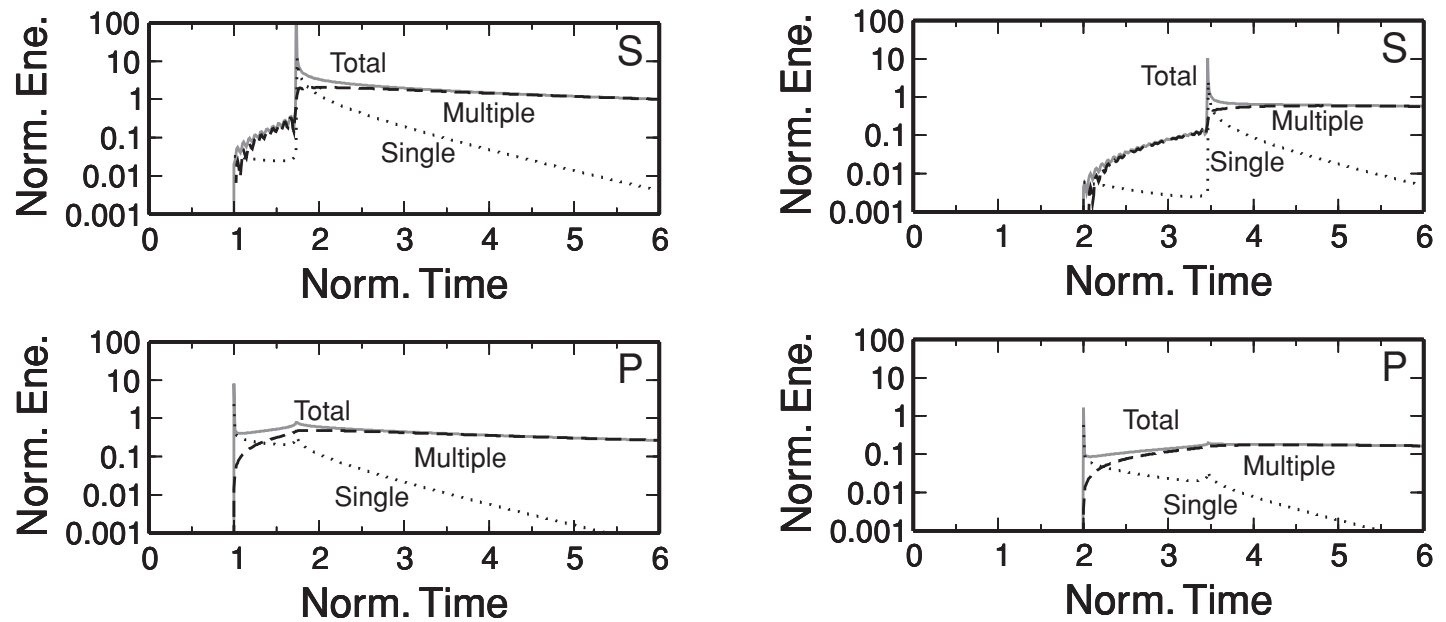

Fig. 3. Contribution of single scattering (dotted curves) and multiple scattering (broken curves) in total energy density (gray solid curves). The receiver is located at a normalized distance of 1 and 2 for the left and the right panels, respectively. $S$ wave and $P$ wave are shown in the top and the bottom panels, respectively. The single-scattering term is dominant around the onset times of a $P$ wave and an $S$ wave. The multiple-scattering term grows soon after the $P$-wave onset.
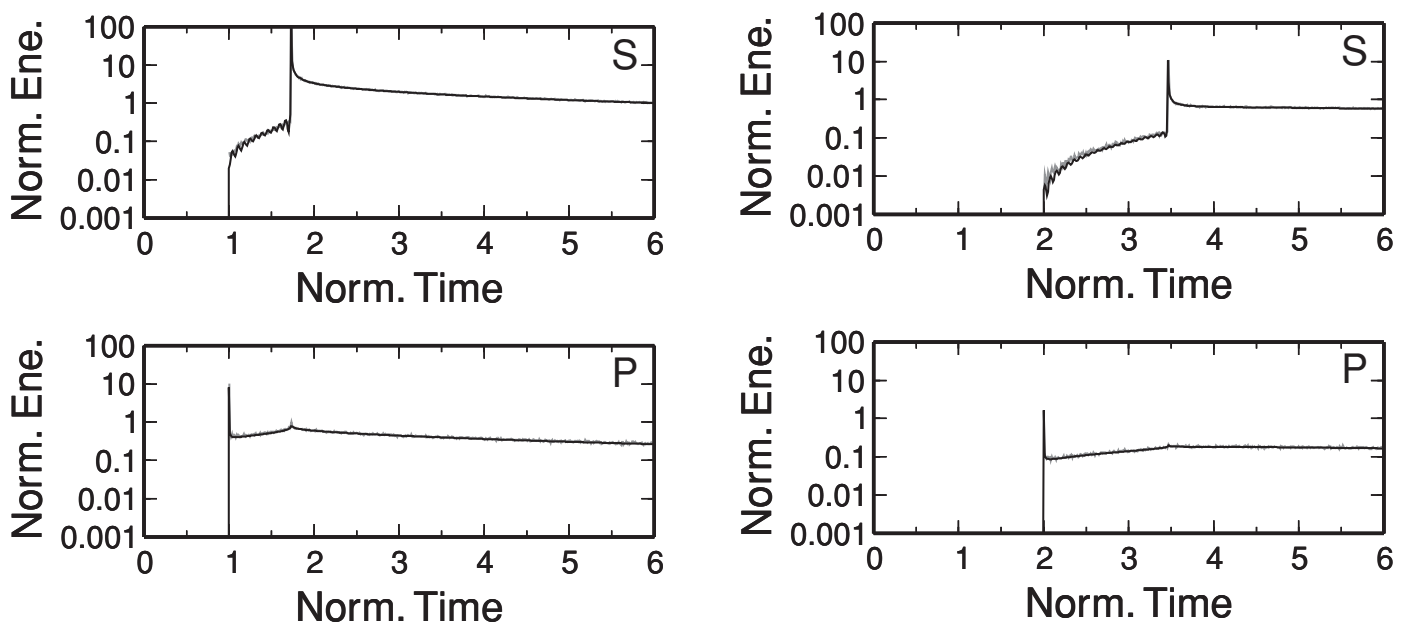

Fig. 4. Temporal changes of $P$-wave and $S$-wave energy. The receiver is located at a normalized distance of 1 and 2 for the left and the right panels, respectively. All of the direct-wave, the single-scattering, and the multiple-scattering terms are included. Solid curves are from our implementation. Gray curves are calculated by the Monte Carlo method for comparison.

practical applications. However, our formulation is useful from the viewpoint of the physics of wave propagation in random media. Our formulation makes it possible to understand directly the effect of dimensionality on the radiative transfer of elastic waves by comparison with formulations for three-dimensional cases. Another benefit of our formulation concerns the validity of seismic interferometry as discussed in this section.

Recently, the partitioning of $P$-wave and $S$-wave energy draws attention to the applicability of seismic interferometry, as shown by Sánchez-Sesma and Campillo (2006). This is also of importance in investigating which is predominant between single scattering and multiple scattering in seismic coda (e.g. Shapiro et al., 2000). Intensive studies have been carried out so far in three-dimensional scattering media (e.g. Margerin et al., 2000). Our formulation enables us to quantitatively investigate the partitioning problem in two-dimensional scattering media, which has scarcely been considered.
In order to solve the problem, we can start from Eqs. (27) and (29). By taking the limit of $t \rightarrow \infty$ after dividing Eq. (29) by Eq. (27), we obtain the $S$-to- $P$ energy ratio in equilibrium:

$$
\lim _{t \rightarrow \infty} \frac{\int_{-\infty}^{\infty} \int_{-\infty}^{\infty} d \mathbf{x} E^{S}(\mathbf{x}, t)}{\int_{-\infty}^{\infty} \int_{-\infty}^{\infty} d \mathbf{x} E^{P}(\mathbf{x}, t)}=\frac{\alpha_{0} g_{0}^{P S}}{\beta_{0} g_{0}^{S P}}=\gamma^{2} .
$$

The relation (A.10) is used in the second equality. Equation (33) is consistent with a result in Sánchez-Sesma and Campillo (2006). For the Poisson solid, the $S$-to- $P$ energy ratio will converge to 3 irrespective of the initial $S$-to$P$ energy ratio at the source. This is thanks to multiple conversion scatterings between a $P$ wave and an $S$ wave. And (A.10) shows that seismic waves tend to become $S$ waves easily through conversion scatterings.

The time necessary to attain a state of equilibrium is sometimes called the equilibration time. We can derive the 

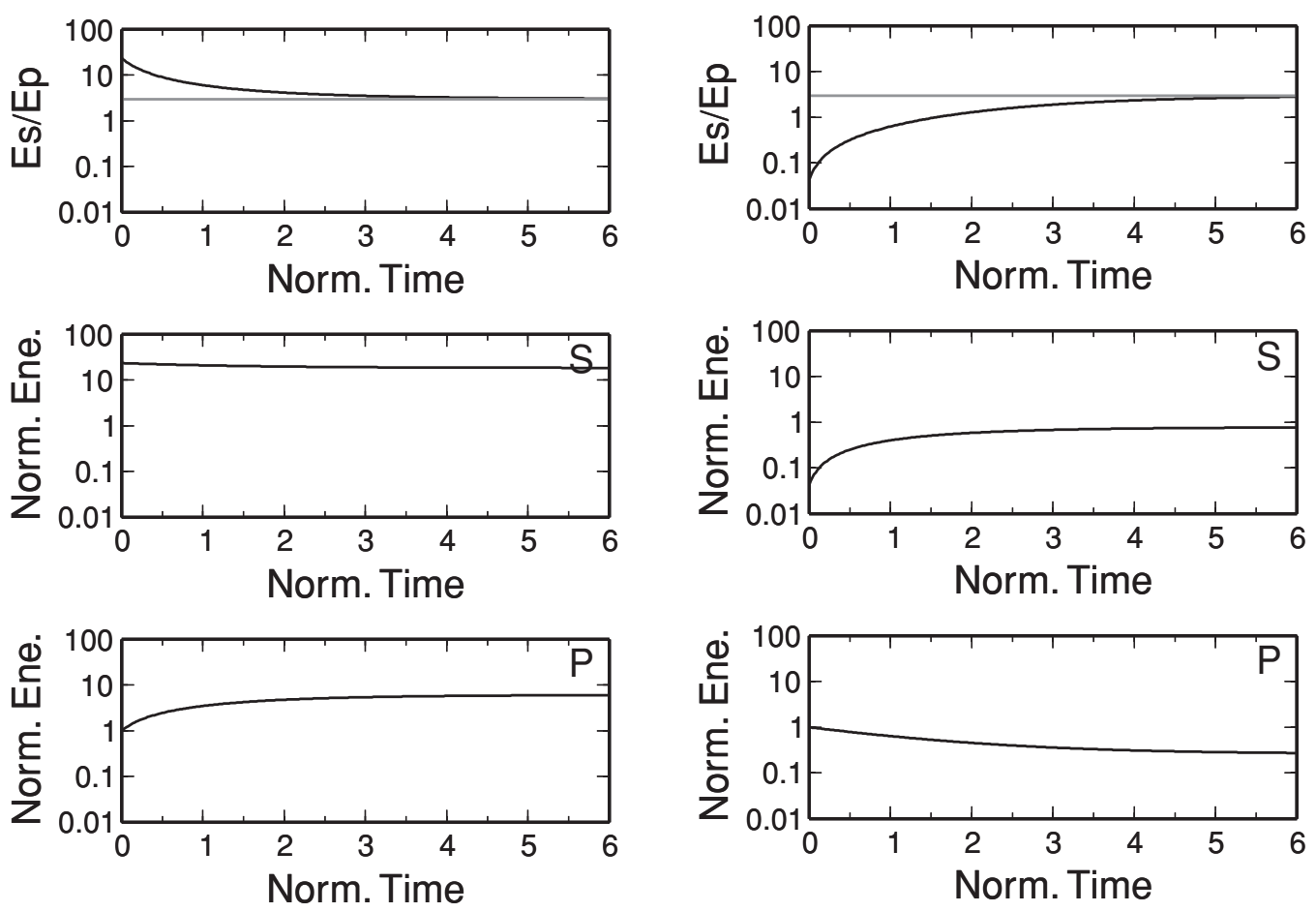

Fig. 5. Temporal change of $P$-wave (bottom panel) and $S$-wave energy (middle panel) in all the space. The top panel shows the $S$-to- $P$ energy ratio. The gray line shows the equilibrated value of 3 which is expected for a two-dimensional Poisson medium at large lapse times. The initial value of the $S$-to- $P$ energy ratio is assumed to be 23.38 and 0.043 for the left and the right panels, respectively.

equilibration time from the time constant of the exponential terms on the right-hand side of Eqs. (27) and (29) for $b=0$ :

$$
t_{\mathrm{eq}}=\left(\alpha_{0} g_{0}^{P S}+\beta_{0} g_{0}^{S P}\right)^{-1}
$$

Equation (34) is found to be the same as the threedimensional cases shown by Sato (1994). In the twodimensional cases, the relation (A.10) holds between the $P$-to- $S$ and the $S$-to- $P$ conversion scattering coefficients. So the equilibration time can be expressed as:

$$
t_{\mathrm{eq}}^{2 \mathrm{D}}=\left[\alpha_{0} g_{0}^{P S}\left(1+\frac{1}{\gamma^{2}}\right)\right]^{-1}=\left[\beta_{0} g_{0}^{S P}\left(1+\gamma^{2}\right)\right]^{-1} .
$$

These expressions are obtained for the two-dimensional case for the first time. Contrasting with Eq. (A.10) for two-dimensional cases, the following relation holds for the three-dimensional cases (e.g. Margerin et al., 2000):

$$
g_{0}^{P S}=2 \gamma^{2} g_{0}^{S P} .
$$

So, in comparison with the two-dimensional case, the equilibration time in the three-dimensional case can be expressed as:

$$
t_{\mathrm{eq}}^{3 \mathrm{D}}=\left[\alpha_{0} g_{0}^{P S}\left(1+\frac{1}{2 \gamma^{3}}\right)\right]^{-1}=\left[\beta_{0} g_{0}^{S P}\left(1+2 \gamma^{3}\right)\right]^{-1} .
$$

The equilibration time is smaller in the two-dimensional case than the three-dimensional case for the same $P$-to$S$ total scattering coefficient $g_{0}^{P S}$. However, this becomes
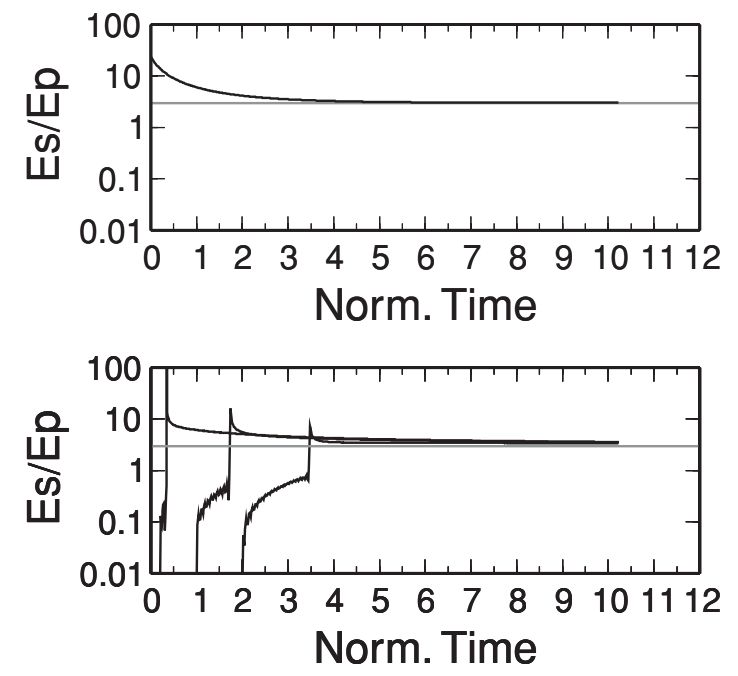

Fig. 6. Temporal change of the $S$-to- $P$ energy ratio in all the space showing the transition to the global equilibrium (top) and those at three receivers with normalized distances of $0.2,1$ and 2 showing the transition into the local equilibrium (bottom). Gray lines show the equilibrated value of 3 which is expected for a two-dimensional Poisson medium at large lapse times.

the opposite for the same $S$-to- $P$ total scattering coefficient $g_{0}^{S P}$. Therefore, the relation depends on the scattering coefficients.

Temporal changes in $P$-wave and $S$-wave energy in all the space are shown in the middle and the bottom panels of Fig. 5. The top panel shows the $S$-to- $P$ energy ratio. The gray line shows the equilibrated value of 3 which is expected for a two-dimensional Poisson medium at large 
lapse times as in Eq. (33). The initial value of the $S$-to- $P$ energy ratio is 23.38 in the left panels. The ratio decreases with time and converges to the expected value of 3 . This is due to the multiple conversion scatterings between a $P$ wave and an $S$ wave. The equilibration time of Eq. (35) is calculated to be 1.5 in the normalized time. In the right panels, we consider a case with a different initial value of the $S$-to- $P$ energy ratio of $0.043(=1 / 23.38)$. For this case, the $S$-to- $P$ energy ratio gradually increases with time and converges to the value of 3 . From these tests, we confirm that the equilibrated value of 3 is irrespective of the initial value at the source and is the result of multiple conversion scatterings between a $P$ wave and an $S$ wave.

In Fig. 6, the bottom panel shows the $S$-to- $P$ energy ratio at three receivers with normalized distances of $0.2,1$ and 2. The top panel shows the $S$-to- $P$ energy ratio in all the space (or global equilibration) for comparison. Different from the global equilibration, it takes a longer time to reach the equilibrium at each point in the space (or local equilibration). At least, more than 10 in normalized time seems necessary for local equilibration. This is explained by the spatial distribution of energy: $S$ waves are dominant close to the source, and $P$ waves are rich close to an expanding propagation front. The local value of the $S$-to- $P$ energy ratio remains larger than 3 near the source for a longer time due to $S$ waves slowly diffusing outward, though the ratio in the entire space (global value) becomes 3 quickly.

\section{Conclusions}

We have formulated the radiative transfer of $P$-wave and $S$-wave energy from an isotropic source in a twodimensional isotropic scattering media. For a stable numerical calculation of seismogram envelopes, we divide energy densities of $P$ waves and $S$ waves into three parts: the direct-wave terms, the single-scattering terms, and the multiple-scattering terms having an order of higher than or equal to 2. The direct-wave terms and the single-scattering terms can be evaluated analytically. Analytical expressions for the single scatterings are obtained in the spacetime domain using elliptic coordinates. Especially, the single conversion scatterings of $P$-to- $S$ and $S$-to- $P$ have been discovered to be represented by a complete elliptic integral of the first kind. Multiple scattering terms can be expressed by double integration with respect to frequency and wavenumber. Discrete wavenumber summation with respect to wavenumber, and FFT with respect to frequency, is adopted. The results based on the numerical implementation of our formulation have been validated by an independent numerical calculation using the Monte Carlo method. Using our formulation, we investigated the equilibration between $P$-wave and $S$-wave energy in a two-dimensional random full space. The equilibrated value of the $S$-to- $P$ energy ratio has been reproduced and the equilibration time has been derived, for the first time, for two-dimensional media. Though our formulation is made for a canonical problem imposing simple assumptions of an isotropic source radiation and an isotropic scattering pattern, it enables us to see the effect of dimensionality on the radiative transfer of elastic waves. It is also helpful in understanding the mathematical background of radiative transfer theory.
Acknowledgments. Constructive comments from the editor Dr. Tatsuhiko Hara and an anonymous reviewer are greatly acknowledged. We are very grateful to the other reviewer, Dr. Jun Kawahara, for pointing out many typing errors left in the original manuscript. We used Generic Mapping Tools (Wessel and Smith, 1998). This study was supported by Grant-in-Aid for Scientific Research (C) (20540413) and for Young Scientists (B) (20740248) from the Japan Society for the Promotion of Science (JSPS) and the Ministry of Education, Culture, Sports, Science and Technology (MEXT). We also thank JSPS and Deutsche Forschungsgemeinschaft (DFG) for support under the Japan-Germany Research Cooperative Program.

\section{Appendix A. Derivation of the Single-Scattering Terms}

We derive the single-scattering terms using the elliptical coordinate (e.g. page 46 in Sato and Fehler, 1998; page 1195 in Morse and Feshbach, 1953). The location of a scatterer $\mathbf{z}=\left(z_{1}, z_{2}\right)^{T}$ is:

$$
\begin{aligned}
& z_{1}= \pm \frac{r}{2} \sqrt{\left(v^{2}-1\right)\left(1-w^{2}\right)} \\
& z_{2}=\frac{r}{2}(1+v w),
\end{aligned}
$$

where the ranges of coordinates are $1 \leq v \leq \infty,-1 \leq$ $w \leq 1$, and the constant $v$ corresponds to an ellipse and the constant $w$ corresponds to a hyperbola. The source is located at the origin $z_{1}=0$ and $z_{2}=0$ or $v=1$, and $w=-1$, and the receiver is located at $z_{1}=0$ and $z_{2}=r$ corresponding to $v=1$ and $w=1$. The source-scatterer distance is $r_{a} \equiv \sqrt{z_{1}^{2}+z_{2}^{2}}=\frac{r}{2}(v+w)$, and the scattererreceiver distance is $r_{b} \equiv \sqrt{z_{1}^{2}+\left(z_{2}-r\right)^{2}}=\frac{r}{2}(v-w)$.

Assuming a geometrical symmetry with respect to $z_{1}=$ 0 , we can derive the $P$-to- $P$ single-scattering term as:

$$
\begin{aligned}
E^{P P, 1}(\mathbf{x}, t) & =\frac{2 W^{P} g_{0}^{P P} e^{-\eta t}}{(2 \pi)^{2} \alpha_{0}} \int_{0}^{\infty} d z_{1} \int_{-\infty}^{\infty} d z_{2} \frac{\delta\left(t-\frac{r_{a}+r_{b}}{\alpha_{0}}\right)}{r_{a} r_{b}} \\
& =\frac{2 W^{P} g_{0}^{P P} e^{-\eta t}}{(2 \pi)^{2} \alpha_{0}} \int_{1}^{\infty} d v \int_{-1}^{1} d w \frac{\delta\left(t-\frac{r v}{\alpha_{0}}\right)}{\sqrt{\left(v^{2}-1\right)\left(1-w^{2}\right)}} \\
& =\frac{W^{P} g_{0}^{P P} e^{-\eta t}}{2 \pi \alpha_{0}} \frac{H\left(\frac{\alpha_{0} t}{r}-1\right)}{\sqrt{t^{2}-\left(\frac{r}{\alpha_{0}}\right)^{2}}} \\
& =\frac{W^{P} g_{0}^{P P} e^{-\eta t}}{2 \pi r} \frac{H\left(\frac{\alpha_{0} t}{r}-1\right)}{\sqrt{\left(\frac{\alpha_{0} t}{r}\right)^{2}-1}}
\end{aligned}
$$

Thus, Eq. (18) is derived. The $S$-to- $S$ single-scattering 
term can be derived similarly as:

$$
\begin{aligned}
E^{S S, 1}(\mathbf{x}, t) & =\frac{W^{S} g_{0}^{S S} e^{-\eta t}}{2 \pi \beta_{0}} \frac{H\left(\frac{\beta_{0} t}{r}-1\right)}{\sqrt{t^{2}-\left(\frac{r}{\beta_{0}}\right)^{2}}} \\
& =\frac{W^{S} g_{0}^{S S} e^{-\eta t}}{2 \pi r} \frac{H\left(\frac{\beta_{0} t}{r}-1\right)}{\sqrt{\left(\frac{\beta_{0} t}{r}\right)^{2}-1}} .
\end{aligned}
$$

Conversion scattering terms need more careful treatment. The $P$-to- $S$ single-scattering term is expressed as:

$$
\begin{aligned}
& E^{P S, 1}(\mathbf{x}, t)=\frac{2 W^{P} g_{0}^{P S} e^{-\eta t}}{(2 \pi)^{2} \beta_{0}} \int_{0}^{\infty} d z_{1} \int_{-\infty}^{\infty} d z_{2} \frac{\delta\left(t-\frac{r_{a}}{\alpha_{0}}-\frac{r_{b}}{\beta_{0}}\right)}{r_{a} r_{b}} \\
& =\frac{2 W^{P} g_{0}^{P S} e^{-\eta t}}{(2 \pi)^{2} \beta_{0}} \int_{1}^{\infty} d v \int_{-1}^{1} d w \frac{\delta\left(t-\frac{r(v+w)}{2 \alpha_{0}}-\frac{r(v-w)}{2 \beta_{0}}\right)}{\sqrt{\left(v^{2}-1\right)\left(1-w^{2}\right)}} .
\end{aligned}
$$

Here, the assumption of Eq. (17) is necessary to take $\exp (-\eta t)$ out of the integral.

We introduce the following quantity

$$
w_{ \pm}=\frac{2 \alpha_{0} t}{r(\gamma-1)} \pm \frac{\gamma+1}{\gamma-1},
$$

which satisfies the equation $\left(\frac{2 \alpha_{0} t}{r(\gamma+1)}-\frac{\gamma-1}{\gamma+1} w\right)^{2}-1=0 . \gamma$ is defined in Eq. (22).

For the evaluation of the integral in (A.5), we need to divide the range of time into two parts. First is the time range where a $P$ wave arrives but an $S$ wave does not arrive. (1) $1 \leq \frac{\alpha_{0} t}{r}<\gamma$

For the range, the following inequality holds: $-1 \leq w \leq$ $w_{-} \leq 1<w_{+}$. According to page 290 in Gradshteyn and Ryzhik (1994), the integral (A.5) can be estimated using the incomplete elliptic integral of the first kind $F(x, y)$ and the complete elliptic integral of the first kind $K(x)$ as

$$
\begin{aligned}
E^{P S, 1} & (\mathbf{x}, t)=\frac{W^{P} g_{0}^{P S} \gamma e^{-\eta t}}{\pi^{2} r(\gamma-1)} \\
& \times \int_{-1}^{w_{-}} d w \frac{1}{\sqrt{\left(w-w_{+}\right)\left(w-w_{-}\right)(1-w)(1+w)}} \\
= & \frac{W^{P} g_{0}^{P S} 2 \gamma e^{-\eta t}}{\pi^{2} r(\gamma-1)} \frac{H\left(\frac{\alpha_{0} t}{r}-1\right)}{\sqrt{2\left(w_{+}-w_{-}\right)}} \\
\times & {\left[F\left(\sin ^{-1} \sqrt{\frac{\left(w_{+}-w_{-}\right)(w+1)}{\left(w_{-}+1\right)\left(w_{+}-w\right)}}, \sqrt{\frac{\left(w_{+}-1\right)\left(w_{-}+1\right)}{2\left(w_{+}-w_{-}\right)}}\right)\right]_{-1}^{w_{-}} } \\
= & \frac{W^{P} g_{0}^{P S} \gamma e^{-\eta t}}{\pi^{2} r} \frac{H\left(\frac{\alpha_{0} t}{r}-1\right)}{\sqrt{\gamma^{2}-1}} F\left(\frac{\pi}{2}, \sqrt{\frac{\left(\alpha_{0} t / r\right)^{2}-1}{\gamma^{2}-1}}\right) \\
= & \frac{W^{P} g_{0}^{P S} \gamma e^{-\eta t}}{\pi^{2} r} \frac{H\left(\frac{\alpha_{0} t}{r}-1\right)}{\sqrt{\gamma^{2}-1}} K\left(\sqrt{\left.\frac{\left(\alpha_{0} t / r\right)^{2}-1}{\gamma^{2}-1}\right)} .\right.
\end{aligned}
$$

(2) $\frac{\alpha_{0} t}{r} \geq \gamma$

For the second range, the following inequality holds: $-1 \leq w \leq 1 \leq w_{-}<w_{+}$. Similarly, according to page 290 in Gradshteyn and Ryzhik (1994), the integral can be estimated:

$$
\begin{aligned}
E^{P S, 1}(\mathbf{x}, t)=\frac{W^{P} g_{0}^{P S} \gamma e^{-\eta t}}{\pi^{2} r(\gamma-1)} \\
\quad \times \int_{-1}^{1} d w \frac{1}{\sqrt{\left(w-w_{+}\right)\left(w-w_{-}\right)(1-w)(1+w)}} \\
=\frac{W^{P} g_{0}^{P S} 2 \gamma e^{-\eta t}}{\pi^{2} r(\gamma-1)} \frac{H\left(\frac{\alpha_{0} t}{r}-1\right)}{\sqrt{\left(w_{+}-1\right)\left(w_{-}+1\right)}} \\
\times\left[F\left(\sin ^{-1} \sqrt{\frac{\left(w_{+}-1\right)(w+1)}{2\left(w_{+}-w\right)}}, \sqrt{\frac{2\left(w_{+}-w_{-}\right)}{\left(w_{+}-1\right)\left(w_{-}+1\right)}}\right)\right]_{-1}^{1} \\
=\frac{W^{P} g_{0}^{P S} \gamma e^{-\eta t}}{\pi^{2} r} \frac{H\left(\frac{\alpha_{0} t}{r}-1\right)}{\sqrt{\left(\alpha_{0} t / r\right)^{2}-1}} F\left(\frac{\pi}{2}, \sqrt{\frac{\gamma^{2}-1}{\left(\alpha_{0} t / r\right)^{2}-1}}\right) \\
=\frac{W^{P} g_{0}^{P S} \gamma e^{-\eta t}}{\pi^{2} r} \frac{H\left(\frac{\alpha_{0} t}{r}-1\right)}{\sqrt{\left(\alpha_{0} t / r\right)^{2}-1}} K\left(\sqrt{\frac{\gamma^{2}-1}{\left(\alpha_{0} t / r\right)^{2}-1}}\right) .
\end{aligned}
$$

Summarizing the two cases, we obtain Eq. (20).

Finally, the $S$-to- $P$ single scattering term is considered.

$$
\begin{aligned}
& E^{S P, 1}(\mathbf{x}, t)=\frac{2 W^{S} g_{0}^{S P} e^{-\eta t}}{(2 \pi)^{2} \alpha_{0}} \int_{0}^{\infty} d z_{1} \int_{-\infty}^{\infty} d z_{2} \frac{\delta\left(t-\frac{r_{a}}{\beta_{0}}-\frac{r_{b}}{\alpha_{0}}\right)}{r_{a} r_{b}} \\
& =\frac{2 W^{S} g_{0}^{S P} e^{-\eta t}}{(2 \pi)^{2} \alpha_{0}} \int_{1}^{\infty} d v \int_{-1}^{1} d w \frac{\delta\left(t-\frac{r(v+w)}{2 \beta_{0}}-\frac{r(v-w)}{2 \alpha_{0}}\right)}{\sqrt{\left(v^{2}-1\right)\left(1-w^{2}\right)}} \\
& =\frac{W^{S} g_{0}^{S P} \beta_{0}}{W^{P} g_{0}^{P S} \alpha_{0}} E^{P S, 1}(\mathbf{x}, t)=\frac{W^{S}}{W^{P} \gamma^{2}} E^{P S, 1}(\mathbf{x}, t) .
\end{aligned}
$$

In the derivation, the following relation is used:

$$
g_{0}^{P S}=\gamma g_{0}^{S P} .
$$

This relation holds for any type of two-dimensional scattering medium due to the reciprocity (e.g. Aki, 1992).

\section{References}

Abramowitz, M. and I. A. Stegun, Handbook of Mathematical Functions with Formulas, Graphs, and Mathematical Tables, Dover, New York, 1970.

Aki, K., Scattering conversions $\mathrm{P}$ to $\mathrm{S}$ versus S to P, Bull. Seismol. Soc. Am., 82, 1969-1972, 1992.

Aki, K. and B. Chouet, Origin of coda waves: source, attenuation, and scattering effects, J. Geophys. Res., 80, 3322-3342, 1975.

Bouchon, M., A simple method to calculate Green's functions for elastic layered media, Bull. Seismol. Soc. Am., 71, 959-971, 1981.

Chandrasekhar, S., Radiative Transfer, Dover, New York, 1960.

Curtis, A., P. Gerstoft, H. Sato, R. Snieder, and K. Wapenaar, Seismic interferometry-turning noise into signal, The Leading Edge, 25, 10821092, doi:10.1190/1.2349814, 2006.

Gradshteyn, I. S. and I. M. Ryzhik, Table of Integrals, Series, and Products (5th edition in English ed. A. Jeffrey), Academic Press, San Diego, 1994. 
Gusev, A. A. and I. R. Abubakirov, Monte-Carlo simulation of record envelope of a near earthquake, Phys. Earth Planet. Inter., 49, 30-36, 1987.

Hoshiba, M., Simulation of multiple-scattered coda wave excitation based on the energy conservation law, Phys. Earth Planet. Inter., 67, 123-136, 1991.

Kopnichev, Y. F., A model of generation of the tail of the seismogram, Doklady Earth Science Sections, 222, 13-15, 1975.

Maeda, T., H. Sato, and M. Ohtake, Synthesis of Rayleigh-wave envelope on the spherical Earth: Analytic solution of the single isotropicscattering model for a circular source radiation, Geophys. Res. Lett., 30, 1286, doi:10.1029/2002GL016629, 2003.

Margerin, L., M. Campillo, and B. V. Van Tiggelen, Monte Carlo simulation of multiple scattering of elastic waves, J. Geophys. Res., 105, 7873-7892, 2000.

Morse, P. M. and H. Feshbach, Methods of Theoretical Physics, Vols. I and II, McGraw-Hill, New York, 1953.

Press, W. H., S. A. Teukolsky, W. T. Vetterling, and B. P. Flannery, Numerical Recipes in FORTRAN, second edition, Cambridge University Press, Cambridge, 1992.

Przybilla, J., M. Korn, and U. Wegler, Radiative transfer of elastic waves versus finite difference simulations in two-dimensional random media, J. Geophys. Res., 111, B04305, doi:10.1029/2005JB003952, 2006.

Ryzhik, L. V., G. C. Papanicolaou, and J. B. Keller, Transport equations for elastic and other waves in random media, Wave Motion, 24, 327-370, 1996.

Sánchez-Sesma, F. J. and M. Campillo, Retrieval of Green's function from cross correlation: The canonical elastic problem, Bull. Seismol. Soc. Am., 96, 1182-1191, 2006.

Sato, H., Energy propagation including scattering effect: Single isotropic scattering approximation, J. Phys. Earth, 25, 27-41, 1977a.

Sato, H., Single isotropic scattering model including wave conversions: Simple theoretical model of the short period body wave propagation, $J$. Phys. Earth, 25, 163-176, 1977b.

Sato, H., Energy transportation in one- and two-dimensional scattering media: Analytic solutions of the multiple isotropic scattering model,
Geophys. J. Int., 112, 141-146, 1993.

Sato, H., Multiple isotropic scattering model including P-S conversions for the seismogram formation, Geophys. J. Int., 117, 487-494, 1994.

Sato, H. and M. Fehler, Seismic Wave Propagation and Scattering in the Heterogeneous Earth, Springer-Verlag, New York, 1998.

Shang, T. and L. Gao, Transportation theory of multiple scattering and its application to seismic coda waves of impulsive source, Scientia Sinica (series B, China), 31, 1503-1514, 1988.

Shapiro, N. M., M. Campillo, L. Margerin, S. K. Singh, V. Kostoglodov, and J. Pacheco, The energy partitioning and the diffusive character of the seismic coda, Bull. Seismol. Soc. Am., 90, 655-665, 2000.

Snieder, R., Coda wave interferometry and the equilibration of energy in elastic media, Phys. Rev. E, 66, 04615, 1-8, 2002.

Trégourès, N. P. and B. A. van Tiggelen, Quasi-two-dimensional transfer of elastic waves, Phys. Rev. E, 66, 036601, 1-16, 2002.

Weaver, R. L., On diffuse waves in solid media, J. Acoust. Soc. Am., 71, 1608-1609, 1982.

Wessel, P. and W. H. F. Smith, New improved version of the Generic Mapping Tools released, Eos Trans. AGU, 79, 579, 1998.

$\mathrm{Wu}, \mathrm{R}$. S., Multiple scattering and energy transfer of seismic wavesseparation of scattering effect from intrinsic attenuation-I. Theoretical modeling, Geophys. J. R. Astron. Soc., 82, 57-80, 1985.

Yamamoto, M. and H. Sato, Multiple scattering and mode conversion revealed by an active seismic experiment at Asama volcano, Japan, $J$. Geophys. Res., doi:10.1029/2009JB007109, 2010 (in press).

Yoshimoto, K., Monte Carlo simulation of seismogram envelopes in scattering media, J. Geophys. Res., 105, 6153-6161, 2000.

Zeng, Y., Theory of scattered P- and S-wave energy in a random isotropic scattering medium, Bull. Seismol. Soc. Am., 83, 1264-1276, 1993.

Zeng, Y., F. Su, and K. Aki, Scattering wave energy propagation in a random isotropic scattering medium 1. Theory, J. Geophys. Res., 96, 607-619, 1991.

H. Nakahara (e-mail: naka@zisin.gp.tohoku.ac.jp) and K. Yoshimoto 